\title{
SPECTRAL MAXIMAL PROJECTIONS AND PROJECTION-RELATIVE DECOMPOSABILITY ON BANACH SPACES
}

\author{
GERARD DUBOIS \\ Institut National des Sciences Appliquées de Lyon, Laboratoire Modélisation Mathématiques de Lyon, \\ Bâtiment Léonard de Vinci, $F$ - 69621 Villeurbanne Cedex \\ e-mail: maths@insa-lyon.fr
}

(Received 15 July, 2003; accepted 7 January, 2004)

\begin{abstract}
We define and study some properties of spectral maximal projections of a bounded operator on a complex Banach space. Then we apply these results to the new concepts of weakly projection-relative decomposable operators and projectionrelative decomposable operators in the spirit of the works of C. Foias [6], A. Jafarian [7], I. Erdelyi and R. Lange [5].
\end{abstract}

2000 Mathematics Subject Classification. 47B40.

1. Introduction. Let $X$ be a complex Banach space, $B(X)$ the algebra of all bounded linear operators on $X$, and $\mathbb{C}$ the field of complex numbers. For an operator $T \in B(X), \sigma(T)$ is the spectrum of $T$ and $\rho(T)=\sigma(T)^{c}$ its resolvent. For $\lambda \in \rho(T)$ we shall use the notation $R(\lambda, T)=(\lambda-T)^{-1}$. When $f$ is an analytic function defined on an open neighborhood of $\sigma(T)$ we can define the bounded operator $f(T)$ on $X$ by

$$
f(T)=\frac{1}{2 \pi i} \int_{\Gamma} f(\lambda) R(\lambda, T) d \lambda,
$$

$\Gamma$ being an admissible contour surrounding $\sigma(T)$. Let $T \in B(X)$. An invariant bounded projection under $T$ is a bounded projection $p$ on $X$ such that $p X$ is invariant for $T$. Invariant subspaces $Y$ of $X$ or invariant bounded projections $p$ produce the restrictions $T \mid Y$ or $T_{p}$ as well as the coinduced operators $T^{Y}$ or $T^{p}$ on the quotient spaces $X / Y$ or $X / p X$. We say that $Y$ or $p$ are $\sigma$-invariant under $T$ if $\sigma(T \mid Y) \subset \sigma(T)$ or $\sigma\left(T_{p}\right) \subset \sigma(T)$ which implies $\sigma(T)=\sigma(T \mid Y) \cup \sigma\left(T^{Y}\right)$ or $\sigma(T)=\sigma\left(T_{p}\right) \cup \sigma\left(T^{p}\right)$. Moreover $Y$ or $p$ are said to be hyperinvariant for $T$ if $Y$ or $p X$ is invariant under each $R \in B(X)$ that commutes with $T . T \in B(X)$ is said to have the single-valued extension property if for every function $f: D \rightarrow X(D$ open in $\mathbb{C})$ analytic on $D$, the condition $(\lambda-T) f(\lambda) \equiv 0$ on $D$ implies $f \equiv 0$. For such an operator, the local resolvent set $\rho_{T}(x)$ is defined for every $x \in X$ and there exists a unique $X$-valued analytic function $\tilde{x}_{T}$ satisfying the equation $(\lambda-T) \tilde{x}_{T}(\lambda)=x$ on $\rho_{T}(x)$. Lastly $X_{T}(F)=\left\{x \in X \mid \sigma_{T}(x) \subset F\right\}$ for a subset $F$ of $\mathbb{C}$.

\section{Spectral maximal projections.}

Definition 2.1. Given $T \in B(X)$, an invariant bounded projection $p$ for $T$ is called a spectral maximal projection of $T$ if for any invariant bounded projection $q$ under $T$, the inclusion $\sigma\left(T_{q}\right) \subset \sigma\left(T_{p}\right)$ implies $q X \subset p X$. 
REMARK 2.2. If $Y$ is a spectral maximal space of $T \in B(X)$ such that $Y$ is complemented in $\mathrm{X}$, then there exists a spectral maximal projection $p$ such that $Y=p X$. In particular, if $X$ is a Hilbert space, the spectral maximal subspaces are exactly the invariant subspaces $Y=p X$ in which $p$ is a spectral maximal projection of $T$.

EXAMPLE 2.3. Let $T$ be a quasispectral operator of class $\Gamma$ with a spectral measure $E\left(\right.$.) of class $\Gamma$, then $X_{T}(F)=E(F) X$ for all closed $F \subset \mathbb{C}[\mathbf{1}$, Lemma 1]. Hence $E(F)$ is a spectral maximal projection of $T$ for each closed $F \subset \mathbb{C}$.

EXAMPLE 2.4. Let $T \in B(X)$ and $\sigma(T)$ be totally disconnected. Let $\delta$ be a separate part of $\sigma(T)$ and $A_{T}(\delta)=\frac{1}{2 \pi i} \int_{\Gamma} R(\lambda, T) d \lambda$ be the spectral projection corresponding to $\delta$, where $\Gamma$ is a system of curves situated in $\rho(T)$ and surrounding $\delta$. Then $A_{T}(\delta)$ is a spectral maximal projection of $T, A_{T}(\delta) X$ being a spectral maximal space of $T$. See [3, Proposition 1.3.10].

THEOREM 2.5. Every spectral maximal projection of $T \in B(X)$ is hyperinvariant under $T$ and $\sigma(T)=\sigma\left(T_{p}\right) \cup \sigma\left(T^{p}\right)$.

Proof. Let $R \in B(X)$ commute with $T$. Then for each $\lambda \in \rho(T), \lambda-R$ is an isomorphism in $B(X)$ commuting with $T$. We can write $(\lambda-R) p X=q X$ where $q$ is the bounded projection defined by $q=(\lambda-R) p(\lambda-R)^{-1}$. From $T_{q}=(\lambda-R) T_{p}(\lambda-R)^{-1}$ it follows that $\sigma\left(T_{q}\right)=\sigma\left(T_{p}\right)$ which implies $q X \subset p X$. Hence $R p X \subset p X$.

TheOREM 2.6. Given $T \in B(X)$, let $f: D \rightarrow \mathbb{C}$ be analytic and injective on an open neighborhood $D$ of $\sigma(T)$. A projection $p$ in $B(X)$ is a spectral maximal projection for $T$ if and only if it is a spectral maximal projection for $f(T)$.

Proof. First we prove the 'if' part of the assertion. Let $q$ be an invariant bounded projection for $T$ that satisfies condition $\sigma\left(T_{q}\right) \subset \sigma\left(T_{p}\right) \subset \sigma(T)$ (the last inclusion is a consequence of the hyperinvariant property of $p$ ). Now we can write

$$
\begin{aligned}
\sigma\left(f(T)_{q}\right) & =\sigma\left(f\left(T_{q}\right)\right) \\
& =f\left(\sigma\left(T_{q}\right)\right) \\
& \subset f\left(\sigma\left(T_{p}\right)\right)=\sigma\left(f\left(T_{p}\right)\right)=\sigma\left(f(T)_{p}\right)
\end{aligned}
$$

and it follows that $q X \subset p X$.

Conversely, let $p$ be a spectral maximal projection of $T$ and let $q$ be an invariant projection under $f(T)$ such that $\sigma\left(f(T)_{q}\right) \subset \sigma\left(f\left(T_{p}\right)\right) \subset \sigma(f(T))$. Then

$$
\begin{aligned}
f\left(\sigma\left(T_{q}\right)\right) & =\sigma\left(f\left(T_{q}\right)\right) \\
& =\sigma\left(f(T)_{q}\right) \\
& \subset \sigma\left(f(T)_{p}\right)=\sigma\left(f\left(T_{p}\right)\right)=f\left(\sigma\left(T_{p}\right)\right),
\end{aligned}
$$

which leads to the desired conclusion.

DeFinition 2.7. For $T \in B(X)$, we say that an invariant bounded projection $p$ under $T$ is $T$-absorbent if, for any $x \in p X$ and all $\lambda \in \sigma\left(T_{p}\right)$, the equation $(\lambda-T) y=x$ has all solutions $y$ in $p X$.

THEOREM 2.8. Given $T \in B(X)$ and $p$ a spectral maximal projection for $T$ then $p$ is T-absorbent. 
Proof. The proof is similar to that of [5, Theorem 3.7] and we shall only sketch it. Let $\lambda \in \sigma\left(T_{p}\right), x \in p X$ and let $y$ be a solution of the equation $(\lambda-T) y=x$. If $y \notin p X$, by putting $Y_{0}=p X \oplus \mathbb{C} y$ we see that $Y_{0}=p_{0} X$ with $p_{0}$ a bounded projection in $B(X)$ invariant under $T$ and from the inclusion $\sigma\left(T_{p_{0}}\right) \subset \sigma\left(T_{p}\right)$ we should have $p_{0} X \subset p X$ which is preposterous.

COROllary 2.9. Let $T \in B(X)$ have the single-valued extension property. If $p$ is a spectral maximal projection for $T$, then $p X$ is analytically invariant for $T$; that is for every function $f: D \rightarrow X$ analytic on some open $D \subset \mathbb{C}$, the condition $(\lambda-T) f(\lambda) \in p X$ implies that $f(\lambda) \in p X$.

Proof. This result is well known when $Y$ is an invariant $T$-absorbing subspace of $X$ and $T$ has the single-valued extension property [5, Theorem 2.26].

\section{Weakly projection-relative decomposable operators.}

Definition 3.1. $T \in B(X)$ is said to be weakly projection-relative (respectively $c$ weakly projection-relative) decomposable if for every open cover $\left\{G_{i}\right\}_{1 \leq i \leq n}$ of $\sigma(T)$, there is a system of spectral maximal projections $\left\{p_{i}\right\}_{1 \leq i \leq n}$ of $T$ (respectively commuting with $T$ ) which performs the following asymptotic spectral decomposition.

1. $\sigma\left(T_{p_{i}}\right) \subset G_{i}$ for every $1 \leq i \leq n$.

2. $X=\overline{\sum_{i=1}^{n} p_{i} X}$.

Proposition 3.2. Let $T$ be weakly projection-relative (respectively c-weakly projection-relative) decomposable. If $G \subset \mathbb{C}$ is open and $G \cap \sigma(T) \neq \emptyset$, then there exists a non zero spectral maximal projection $p$ (respectively commuting with $T$ ) with the property $\sigma\left(T_{p}\right) \subset G$.

Proof. Let $G^{\prime}$ be a second open set such that $\left\{G, G^{\prime}\right\}$ is a covering of $\sigma(T), \sigma(T) \not \subset G^{\prime}$. Then there are $p, q$ spectral maximal projections of $T$ satisfying $\sigma\left(T_{p}\right) \subset G, \sigma\left(T_{q}\right) \subset G^{\prime}$, $X=\overline{p X+q X}$. Now if $p=0$, we should have $X=q X$ in contradiction with the choice of $G^{\prime}$.

LEMMA 3.3. If $p$ is a spectral maximal projection of an operator $T$ in $B(X)$ and $D$ is a domain such that there is a nonzero analytic $X$-valued function $f$ satisfying the equation $(\lambda-T) f(\lambda)=0$ on $D$, then $D \cap \sigma\left(T_{p}\right)=\emptyset$ or $D \subset \sigma_{\text {point }}\left(T_{p}\right)$, where $\sigma_{\text {point }}\left(T_{p}\right)$ is the point spectrum of $T_{p}$.

Proof. We shall follow the proof of [5, Lemma 6.3], where the key point is the finite dimensional property of the linear manifold $X_{n}=\bigvee\left\{f\left(\lambda_{0}\right), f^{\prime}\left(\lambda_{0}\right), \ldots, f^{(n)}\left(\lambda_{0}\right)\right\}$ which is complemented in $X$ so that we can associate with $X_{n}$ a bounded projection $p_{n}$ invariant under $T$ such that $X_{n}=p_{n} X$.

THEOREM 3.4. Every weakly projection-relative decomposable operator has the single valued extension property.

Proof. Let $T$ be weakly projection-relative decomposable and $f: D \rightarrow X$ be analytic and satisfy the equation $(\lambda-T) f(\lambda)=0$ on an open set $D \subset \mathbb{C}$. We may assume that $D \cap \sigma(T) \neq \varnothing$ and $D$ is a domain. By Proposition 3.2, there is a nonzero spectral maximal projection $p$ of $T$ such that $\sigma\left(T_{p}\right) \subset D$. If $f \neq 0$ on $D$ then, by Lemma 3.3, $D \subset \sigma\left(T_{p}\right)$, which gives a contradiction, $D$ being open and not void. 
TheOREM 3.5. Given $T \in B(X)$, let $f: \mathbb{D} \rightarrow \mathbb{C}$ be analytic and injective on an open neighbourhood $D$ of $\sigma(T)$. Then $T$ is weakly projection-relative (respectively c-projection relative) decomposable if and only if $f(T)$ is.

Proof. Let $f(T)$ be weakly projection-relative decomposable and $\left\{G_{i}\right\}_{1 \leq i \leq n}$ be an open covering of $\sigma(f(T))$. Since $\sigma(T) \subset D$ the sets $G_{i}^{\prime}=G_{i} \cap D, 1 \leq i \leq n$, also form an open covering of $\sigma(T)$. In addition $\left\{f\left(G_{i}^{\prime}\right)\right\}_{1 \leq i \leq n}$ is an open covering of $\sigma(f(T))$ and we can find spectral maximal projections $p_{i}$ of $f(T)$ such that

$$
\begin{aligned}
\sigma\left(f(T)_{p_{i}}\right) & \subset \frac{f\left(G_{i}^{\prime}\right) \quad}{X}=\sum_{i=1}^{n} p_{i} X .
\end{aligned}
$$

But $p_{i}(1 \leq i \leq n)$ are also spectral maximal projections of $T$ by Theorem 2.5 and the inclusion $f\left(\sigma\left(T_{p_{i}}\right)\right) \subset f\left(G_{i}^{\prime}\right)$ leads to

$$
\sigma\left(T_{p_{i}}\right) \subset G_{i}^{\prime} \subset G_{i} \quad(1 \leq i \leq n)
$$

Thus $T$ is weakly projection-relative decomposable. Now, if $p_{i}$ commutes with $f(T)$, then $p_{i}$ commutes with $T$ too. Conversely, the proof is similar.

\section{Projection-relative decomposable spectrum.}

Definition 4.1. $T \in B(X)$ is said to have projection-relative (respectively $c$ projection relative) decomposable spectrum if for every open covering $\left\{G_{i}\right\}_{1 \leq i \leq n}$ of $\sigma(T)$, there is an asymptotic projection-relative decomposition induced by a system $\left\{p_{i}\right\}_{1 \leq i \leq n}$ of spectral maximal projections of $T$ (respectively commuting with $T$ ) such that

1. $\sigma\left(T_{p_{i}}\right) \subset G_{i} \quad(1 \leq i \leq n)$,

2. $X=\overline{\sum_{i=1}^{n} p_{i} X}$,

3. $\sigma(T)=\bigcup_{i=1}^{n} \sigma\left(T_{p_{i}}\right)$.

THEOREM 4.2. Let $T$ be a weakly projection-relative (respectively c-projectionrelative) decomposable operator. The following statements are equivalent.

(i) $T$ has projection-relative (respectively c-projection-relative) decomposable spectrum.

(ii) If $F \subset \sigma(T)$ is closed and $G \supset F$ is open, then there exists a spectral maximal projection $p$ of $T$ (respectively commuting with $T$ ) such that $F \subset \sigma\left(T_{p}\right) \subset G$.

(iii) Every system $\left\{p_{i}\right\}_{1 \leq i \leq n}$ of spectral maximal projections (respectively commuting with $T)$ satisfies $\sigma(T)=\bigcup_{i=1}^{n} \sigma\left(T_{p_{i}}\right)$ whenever $X=\overline{\sum_{i=1}^{n} p_{i} X}$.

Proof. Obviously (iii) implies (i). We shall prove that (i) $\Rightarrow$ (ii). For this, let $F \subset \sigma(T)$ be closed and $G \supset F$ be open. Then $\left\{G, F^{c}\right\}$ is an open covering of $\sigma(T)$ and so there are spectral maximal projections $p, q$ of $T$ satisfying conditions $\sigma\left(T_{p}\right) \subset G, \sigma\left(T_{q}\right) \subset$ $F^{c}, \sigma(T)=\sigma\left(T_{p}\right) \cup \sigma\left(T_{q}\right)$. Consequently $F \subset \sigma\left(T_{p}\right) \subset G$. It remains to prove that (ii) $\Rightarrow$ (iii). Let $\left\{p_{i}\right\}_{1 \leq i \leq n}$ be an arbitrary system of spectral maximal projections of $T$ performing the decomposition $X=\overline{\sum_{i=1}^{n}} p_{i} X$. If $F=\bigcup_{i=1}^{n} \sigma\left(T_{p_{i}}\right) \neq \sigma(T)$ then there 
exists a spectral maximal projection $q$ of $T$ such that $F \subset \sigma\left(T_{q}\right) \neq \sigma(T)$. Now we have

$$
\sigma(T)=\sigma\left(T / \overline{\sum_{i=1}^{n} p_{i} X}\right) \subset \bigcup_{i=1}^{n} \sigma\left(T_{p_{i}}\right) \subset \sigma\left(T_{q}\right) .
$$

It follows that $X=q X$ and $\sigma(T)=\sigma\left(T_{q}\right)$ which is preposterous.

\section{Projection-relative quasi decomposable operators.}

DeFINITION 5.1. A weakly projection-relative (respectively $c$-projection relative) decomposable operator is said to be projection-relative (respectively c-projection relative) quasi decomposable if $X_{T}(F)$ is closed whenever $F \subset \mathbb{C}$ is closed.

THEOREM 5.2. Every projection-relative (respectively c-projection-relative) quasi decomposable operator has projection-relative (respectively c-projection-relative) decomposable spectrum.

Proof. Let $\left\{G_{i}\right\}_{1 \leq i \leq n}$ be a finite open covering of $\sigma(T)$ and let $\left\{p_{i}\right\}_{1 \leq i \leq n}$ be a system of spectral maximal projections of $T$ such that $\sigma\left(T_{p_{i}}\right) \subset G_{i}$ for $1 \leq i \leq n$ and $X=\overline{\sum_{i=1}^{n} p_{i} X}$. If $F=\bigcup_{i=1}^{n} \sigma\left(T_{p_{i}}\right)$ is proper in $\sigma(T)$, then $X_{T}(F)$ is proper in $X$, but each $p_{i} X$ is contained in $X_{T}(F)$, which is preposterous.

THEOREM 5.3. If $T$ is a weakly c-projection-relative decomposable operator, then $T$ is in fact a c-projection-relative quasi decomposable operator.

Proof. Let $F$ be a closed set in $\mathbb{C}$, and $G$ any open set containing $F$. Since $\left\{F^{c}, G\right\}$ is an open covering of $\sigma(T)$, there exist $p_{1}$ and $p_{2}$ spectral maximal projections of $T$ such that $\sigma\left(T_{p_{1}}\right) \subset F^{c}, \sigma\left(T_{p_{2}}\right) \subset G, X=\overline{p_{1} X+p_{2} X}$, with $p_{1}, p_{2}$ commuting with $T$. If $x \in X_{T}(F)$, there exist $x_{1, n} \in p_{1} X, x_{2, n} \in p_{2} X$ such that $x=\lim _{n \rightarrow \infty}\left(x_{1, n}+x_{2, n}\right)$. Now $p_{1} T=T p_{1}$ and so

$$
\begin{aligned}
\sigma_{T}\left(p_{1} x\right) & \subset \sigma_{T}(x) \cap \sigma\left(T_{p_{1}}\right) \\
& \subset F \cap F^{c}=\emptyset .
\end{aligned}
$$

This implies that

$$
\begin{aligned}
p_{1} x=0 & =\lim _{n \rightarrow \infty}\left(p_{1} x_{1, n}+p_{1} x_{2, n}\right) \\
& =\lim _{n \rightarrow \infty}\left(x_{1, n}+p_{1} x_{2, n}\right)
\end{aligned}
$$

and so

$$
\begin{aligned}
x & =\lim _{n \rightarrow \infty}\left(x_{1, n}+x_{2, n}\right)-\lim _{n \rightarrow \infty}\left(x_{1, n}+p_{1} x_{2, n}\right) \\
& =\lim _{n \rightarrow \infty}\left(x_{2, n}-p_{1} x_{2, n}\right) .
\end{aligned}
$$

Since spectral maximal projections are hyperinvariant we have $p_{1} x_{2, n} \in p_{2} X$ and $x \in p_{2} X$. Finally, we obtain

$$
\begin{aligned}
X_{T}(F) & \subset p_{2} X \\
& \subset X_{T}\left(\sigma\left(T_{p_{2}}\right)\right) \\
& \subset X_{T}(G),
\end{aligned}
$$


$G$ being any open set containing $F$. We have

$$
\begin{aligned}
X_{T}(F) & \subset p_{2} X \\
& \subset \bigcap_{F \subset G} X_{T}(G)=X_{T}\left(\bigcap_{F \subset G} G\right)=X_{T}(F) .
\end{aligned}
$$

Thus $X_{T}(F)=p_{2} X$ is closed.

\section{Projection-relative decomposable operators.}

DEFINITION 6.1. $T \in B(X)$ is called projection-relative (respectively c-projectionrelative) decomposable, if for every open covering $\left\{G_{i}\right\}_{1 \leq i \leq n}$ of $\sigma(T)$, there exists a system $\left\{p_{i}\right\}_{1 \leq i \leq n}$ of spectral maximal projections of $T$ (respectively commuting with $T$ ) yielding the following spectral decomposition.

1. $\sigma\left(T_{p_{i}}\right) \subset G_{i}$ for $1 \leq i \leq n$.

2. $X=\sum_{i=1}^{n} p_{i} X$.

REMARK 6.2. Clearly such an operator is projection-relative quasidecomposable and has projection-relative decomposable spectrum.

EXAMPLE 6.3. If $X$ is a Hilbert space, the concepts of projection-relative decomposable operators and decomposable operators are the same.

EXAMPLE 6.4. Let $T$ be a compact operator on $X$ (or more generally an operator with totally disconnected spectrum). Then $T$ is $c$-projection-relative decomposable. To see this, let $\left\{G_{i}\right\}_{1 \leq i \leq n}$ be a finite open covering of $\sigma(T)$, we can choose open-andclosed subsets $\delta_{i}$ of $\sigma(T)$ such that $\delta_{i} \subset G_{i}$ for $1 \leq i \leq n$ and leading to a system $\left\{A_{T}\left(\delta_{i}\right)\right\}_{1 \leq i \leq n}$ of spectral maximal projections commuting with $T$ and which yields $\sigma\left(T_{A_{T}\left(\delta_{i}\right)}\right)=\delta_{i} \subset G_{i}$ for $1 \leq i \leq n$ and $X=\sum_{i=1}^{n} A_{T}\left(\delta_{i}\right) X$.

EXAMPLE 6.5. Quasispectral operators of class $\Gamma$ on $X$ (in Albrecht's sense [1]) with spectral measure $E($.$) of class \Gamma$ are projection-relative decomposable operators. In order to prove this, let us take a finite open covering $\left\{G_{i}\right\}_{1 \leq i \leq n}$ of $\sigma(T)$. Then there exists a finite open covering $\left\{\omega_{i}\right\}_{1 \leq i \leq n}$ of $\sigma(T)$ with $\overline{\omega_{i}} \subset G_{i}$ for every $1 \leq i \leq n$. Put $s_{1}=\omega_{1}$ and $s_{i}=\omega_{i}-\bigcup_{j<i} \omega_{j}$ for $1 \leq i \leq n$. We obtain a finite disjoint covering $\left\{s_{i}\right\}_{1 \leq i \leq n}$ of $\sigma(T)$ by Borel sets. The bounded projections $E\left(\bar{s}_{i}\right)$ form a system of spectral maximal projections of $T$ such that

$$
\begin{aligned}
& \sigma\left(T_{E\left(\bar{s}_{i}\right)}\right) \subset \bar{s}_{i} \subset \bar{\omega}_{i} \subset G_{i} \quad(1 \leq i \leq n) \quad \text { and } \\
& X=E(\sigma(T)) X=\sum_{i=1}^{n} E\left(s_{i}\right) X=\sum_{i=1}^{n} E\left(\bar{s}_{i}\right) X .
\end{aligned}
$$

EXAMPLE 6.6. Prespectral operators of class $\Gamma$ on $X$ and hence spectral operators are $c$-projection-relative decomposable operators. This results from the commutativity property of $T$ and $E$. See [4].

THEOREM 6.7. Let $T \in B(X)$ and $f: D \rightarrow X$ be an analytic injective function on an open neighborhood $D$ of $\sigma(T)$. Then $f(T)$ is projection-relative (c-projection-relative) decomposable if and only if $T$ is. 
Proof. This is similar to that of Theorem 3.5.

Proposition 6.8. Let $T$ be projection-relative decomposable and $p$ be a spectral maximal projection of $T$. Then we have $\sigma\left(T^{p}\right)=\overline{\sigma(T)-\sigma\left(T_{p}\right)}$.

Proof. Suppose that there is $\lambda \in \sigma\left(T^{p}\right)-\overline{\sigma(T)-\sigma\left(T_{p}\right)}$. Then we can find an open covering $\left\{G_{1} \cup G_{2}\right\}$ of $\sigma(T)$ such that

$$
\begin{aligned}
\lambda \notin G_{1} & \supset \overline{\sigma\left(T-\sigma\left(T_{p}\right)\right)}, \\
G_{2} & \cap \overline{\sigma(T)-\sigma\left(T_{p}\right)}=\emptyset .
\end{aligned}
$$

Let $\left\{p_{1}, p_{2}\right\}$ be the spectral maximal projections of $T$ corresponding to this covering of $\sigma(T)$. From the inclusion $\sigma\left(T_{p_{2}}\right) \subset G_{2} \cap \sigma(T) \subset \sigma\left(T_{p}\right)$ we have $p_{2} X \subset p X$. Now let $\dot{x} \in X / p X$ such that $\left(\lambda-T^{p}\right) \dot{x}=0$. If $x \in \dot{x}$ and $x_{1} \in p_{1} X, x_{2} \in p_{2} X$ satisfy $x=x_{1}+x_{2}$, one obtains $(\lambda-T) x_{1}=(\lambda-T) x-(\lambda-T) x_{2} \in p X \cap p_{1} X$, a subspace of $X$ invariant under $\left(\lambda-T_{p_{1}}\right)^{-1}$ (which exists because $\sigma\left(T_{p_{1}}\right) \subset G_{1}$ and $\left.\lambda \notin G_{1}\right)$. It follows that $x_{1}=\left(\lambda-T_{p_{1}}\right)^{-1}(\lambda-T) x_{1} \in p_{1} X \cap p X$ and $\dot{x}=\dot{x}_{1}+\dot{x_{2}}=0$. Hence $\lambda-T^{p}$ is one to one. Now if we take $\dot{y} \in X \mid p X$ and $y=y_{1}+y_{2} \in \dot{y}, y_{1} \in p_{1} X, y_{2} \in p_{2} X$ we can find $x_{1} \in p_{1} X$ such that $\left(\lambda-T_{p_{1}}\right) x_{1}=y_{1}$ (remember that $\left.\lambda \notin G_{1}\right)$. Consequently one obtains $\dot{y}=\dot{y_{1}}=\overline{\left(\lambda-T_{p_{1}}\right) x_{1}}=\overline{(\lambda-T) x_{1}}=\left(\lambda-T^{p}\right) \dot{x_{1}}$ which means that $\left(\lambda-T^{p}\right)$ maps $X / p X$ onto $X / p X$ so that $\lambda \notin \sigma\left(T^{p}\right)$ which is preposterous. The opposite inclusion follows from the hyperinvariant property of $p$.

\section{REFERENCES}

1. E. Albrecht, On some classes of generalized spectral operators, Arch. Math. (Basel) 30 (1978), 297-303.

2. C. Apostol, Spectral decompositions and functional calculus, Rev. Roum. Math. Pures Appl. 13 (1968), 1481-1528. 1968).

3. I. Colojoara and C. Foias, Theory of generalized spectral operators (Gordon and Breach,

4. H. R. Dowson, Spectral theory of linear operators (Academic Press, 1978).

5. I. Erdelyi and R. Lange, Spectral decompositions on Banach spaces, Lecture Notes in Mathematics No. 623 (Springer-Verlag, 1977).

6. C. Foias, Spectral maximal spaces and decomposable operators in Banach spaces, Arch. Math. 14 (1963), 341-349.

7. A. Jafarian, Weak and quasi-decomposable operators, Rev. Roum. Math. Pures Appl. 22 (1977), 195-212.

8. K. Tanahashi and T. Yoshino, A characterization of spectral operators on Hilbert spaces, Proc. Amer. Math. Soc. 90 (1984), 567-570. 